\title{
Masson's Tumour: A Rare Cause of Cervical Mass
}

\author{
Mariana da Silva Leal, Carolina Amado, Bárbara Paracana, Gisela Gonçalves, Mariana Sousa \\ Internal Medicine Department, Centro Hospitalar do Baixo Vouga, Aveiro, Portugal
}

Doi: 10.12890/2021_003078 - European Journal of Case Reports in Internal Medicine - ๑ EFIM 2021

Received: 23/11/2021

Accepted: 05/12/2021

Published: $27 / 12 / 2021$

How to cite this article: da Silva Leal M, Amado C, Paracana B, Gonçalves G, Sousa M. Masson's tumour a rare case of cervical mass. EJCRIM 2021;8: doi:10.12890/2021_003078.

Conflicts of Interests: The authors declare there are no competing interests.

This article is licensed under a Commons Attribution Non-Commercial 4.0 License

\section{ABSTRACT}

Masson's tumour, also known as intravascular papillary endothelial hyperplasia, is a rare non-neoplastic lesion of vascular origin, caused by an excessive reactive proliferation of endothelial cells in normal blood vessels or vascular malformations. It can affect any part of the body, presenting most frequently in the vessels of the head, neck and upper extremities. The authors describe the case of a 76-year-old female patient presenting an anterior cervical mass, measuring $2 \times 2 \mathrm{~cm}$, which was mobile, tender and slightly painful with no alteration of the overlying skin. Complementary study with ultrasound and computed tomography was inconclusive. Total excision of the lesion was performed with histology compatible with intravascular papillary endothelial hyperplasia.

\section{LEARNING POINTS}

- Masson's tumour is a rare and indolent non-neoplastic lesion of vascular origin, that can be easily confused with other pathological entities and misdiagnosed.

- Diagnosis can be a challenge; histological characterization is essential for differentiation from other lesions, such as angiosarcoma and Kaposi's sarcoma.

- Masson's tumour should be considered in patients presenting a single cervical mass.

\section{KEYWORDS}

Cervical mass, intravascular papillary endothelial hyperplasia, Masson's tumor, neck mass

\section{INTRODUCTION}

Masson's tumour, also known as intravascular papillary endothelial hyperplasia (IPEH), is a rare, presumably reactive, non-neoplastic lesion of vascular origin, caused by an excessive reactive proliferation of endothelial cells in normal blood vessels or vascular malformations ${ }^{[1-3]}$. It can affect any part of the body but most frequently involves the vessels of the head, neck and upper extremities ${ }^{[1]}$. Although it has an excellent prognosis, diagnosis can be challenging but is essential, as clinically it can be similar to other benign and malignant conditions that require additional interventions, such as angiosarcoma and Kaposi's sarcoma ${ }^{[4]}$.

\section{CASE DESCRIPTION}

The authors describe the case of a 76-years-old female patient with a medical history of type 2 diabetes mellitus (under oral antidiabetics), dyslipidaemia, supplemented hypothyroidism and overweight. She attended the emergency department complaining of a painful nontraumatic left cervical swelling of 5 days' duration. She denied fever, weight loss, anorexia, night sweats, dysphonia, dysphagia, dyspnoea and other respiratory or constitutional symptoms. There was no history of recent infections, antibiotic therapy or any other relevant epidemiological context. On physical examination, she had an anterior-inferior left-sided raised cervical mass, measuring $2 \times 2 \mathrm{~cm}$, which was 
mobile, tender, slightly painful on palpation, non-pulsatile, and without alterations of the overlying skin. Ganglia chains and the remaining physical examination were normal. A complete blood count, peripheral blood smear and coagulation tests showed no alterations; the erythrocyte sedimentation rate, electrophoretic pattern and C-reactive protein were normal. Bartonella, viral serologies (Toxoplasma gondii, rubella, cytomegalovirus, Epstein-Barr, hepatitis B and C, and HIV) and interferon-gamma release assays were negative. Ultrasound of soft tissues revealed an ovoid mixed nature mass formation, which was hypoechogenic but heterogeneous, measured $26 \times 14$ mm, was close to the external jugular vessels, and had no significant internal vascularization in the Doppler study (Fig. 1). Given the possibility of an abscess collection, empiric antibiotic therapy with cefuroxime and metronidazole was initiated. There was no clinical or ultrasound improvement after 1 week, at which time computed tomography was performed and showed a well-defined rounded formation in the transition to the supraclavicular region, posterior to the external jugular vein, measuring $23 \times 20 \mathrm{~mm}$, with soft tissue density and mild late peripheral enhancement, with apparent calcifications on the lateral side (Fig. 2).

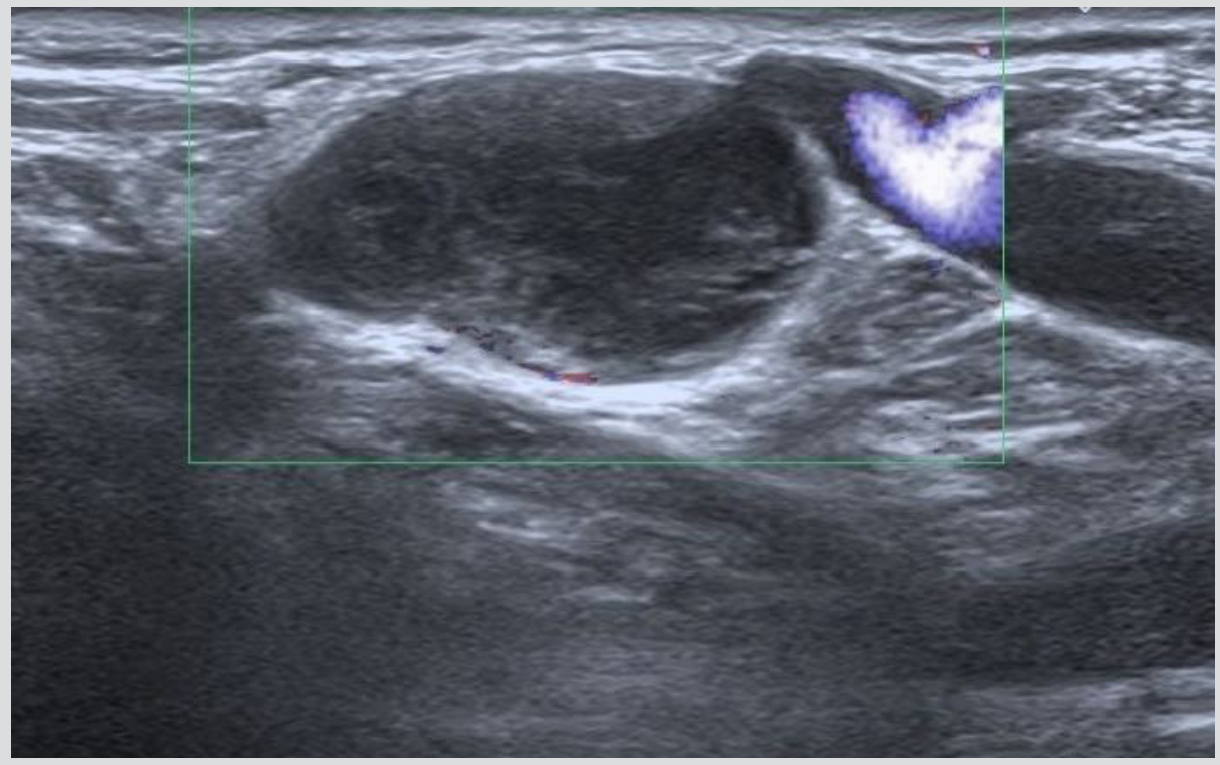

Figure 1. Cervical ultrasound with colour Doppler showing an ovoid hypoechogenic soft tissue mass, with a heterogeneous internal structure and some cystic areas, measuring $26 \times 14 \mathrm{~mm}$. It is adjacent to the external jugular vein, which preserved internal flow

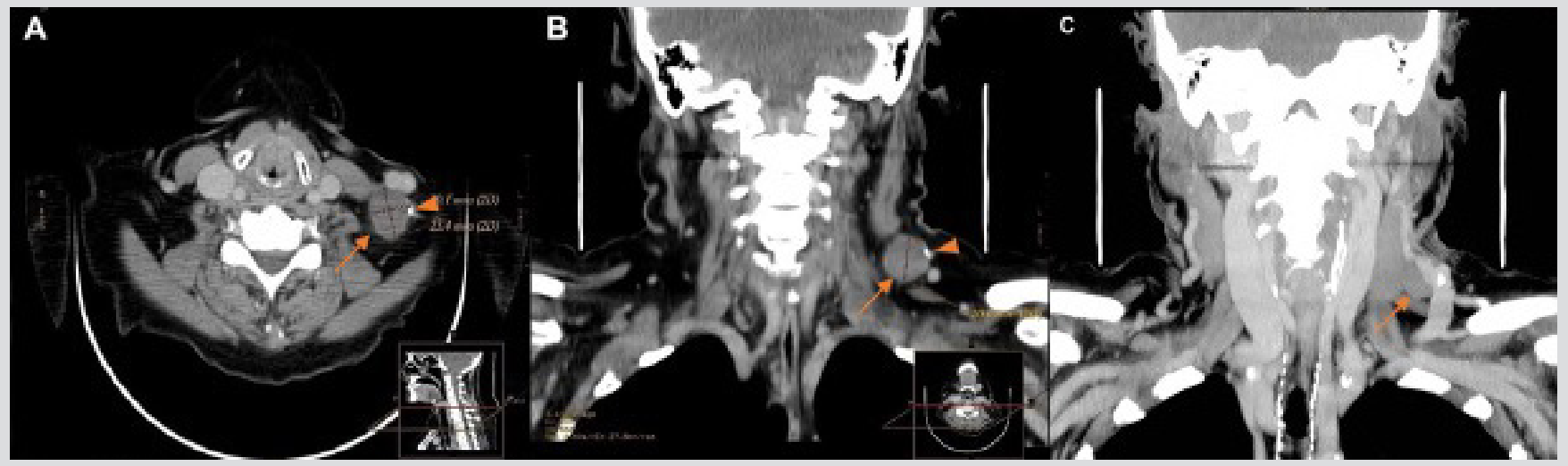

Figure 2. Cervical contrast-enhanced CT shows a well-defined soft tissue cervical mass (arrow) measuring $23 \times 20 \times 20 \mathrm{~mm}$, close to the left external jugular vein, with peripheric calcification (arrow head). (A) axial slice; (B) coronal slice; (C) coronal maximum intensity projection (MIP) reconstruction

Although there were no lesions in the different territories studied, the hypothesis of a probable lymphadenopathy/lymphoproliferative disorder was raised, and excision of the lesion was performed for characterization. It revealed a poorly encapsulated mass with haematic content that ruptured during the procedure. Immunophenotyping (with peripheral blood contamination) showed no alterations of B and T cells. Direct examination and culture of mycobacteria were negative, as were other bacterial and fungal cultures. Histopathology of the lesion presented an incomplete appearance (compatible with rupture), but the lesion was well delimited at the periphery, appearing 
to be surrounded by a fibrous pseudocapsule (Fig. 3A); fibrohistiocytic proliferation (without granulomas) delimited by a fibrous capsule was evident, with associated lymphocytic infiltrate, focal eosinophil and neutrophil infiltration, and angiogenesis-type granulation tissue. This proliferation delimited a central area with packed red blood cells and fibrin, without clear thrombus formation, suggesting central fibrinolysis. Underlying the capsule, some slit-like spaces with endothelial lining were identified, suggesting an intravascular lesion and, multifocally, formation of papillae lined by endothelial cells (Fig. 3B,C) with no evidence of pleomorphic endothelial cells (nor atypia or anaplasia), mitotic figures or necrosis (Fig. 3D), consistent with intravascular papillary endothelial hyperplasia. In the sparse surrounding adipose tissue, cytosteatonecrosis lesions were observed, suggesting previous trauma. There were no post-surgery complications and no evidence of recurrence after 1 year.

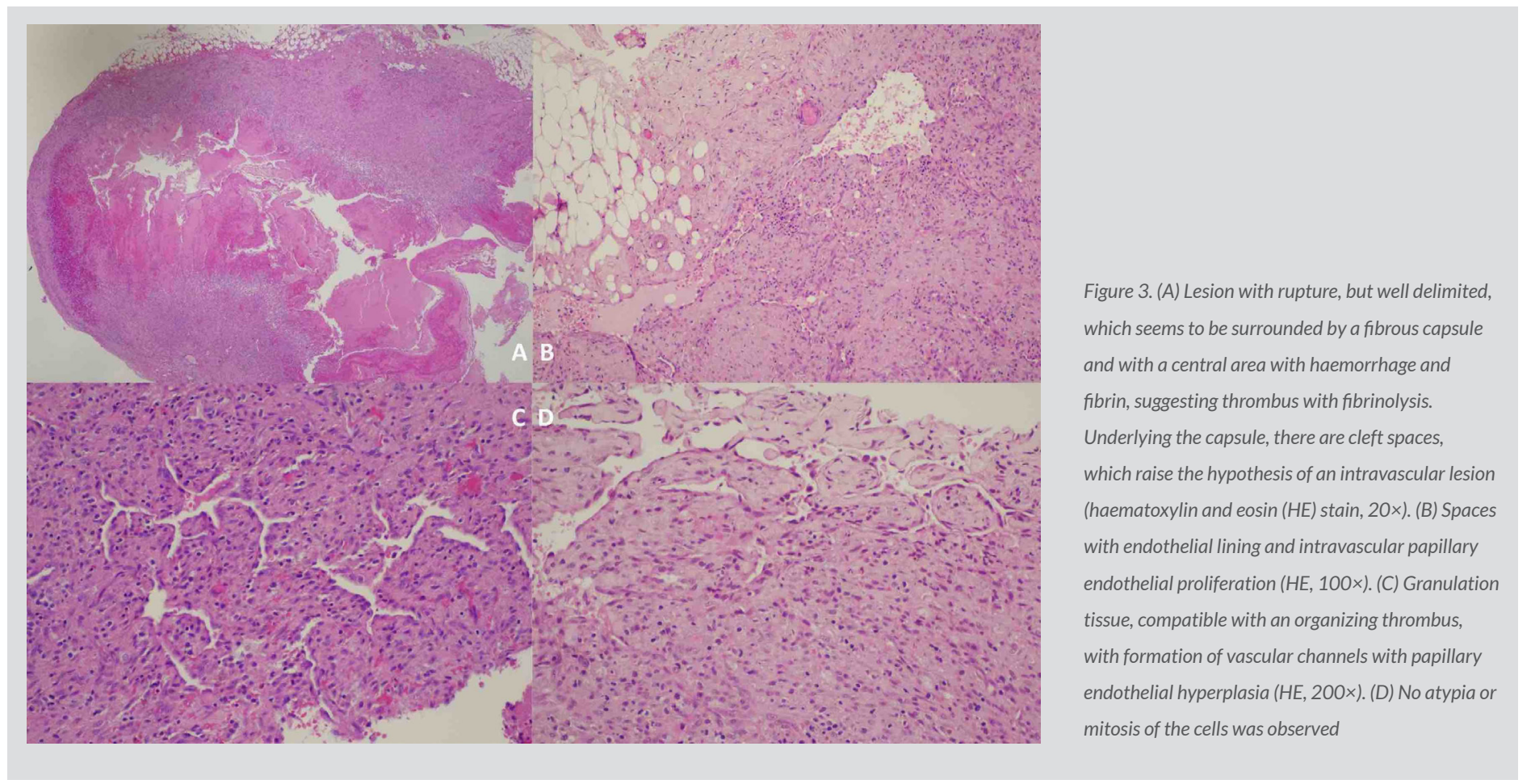

\section{DISCUSSION}

IPEH was first described in 1923 by Pierre Masson and termed 'hemangioendotheliome vegetant intravasculaire', after the identification of an intravascular lesion with unusual proliferative histological behaviour in an infected haemorrhoidal plexus of a 68-year-old man ${ }^{[1]}$. Most lesions described in the literature have involved the head and extremities. However, to the authors' knowledge, IPEH presenting as a neck mass is uncommon, with less than 10 cases described in the literature, with only two close to the external jugular vein ${ }^{[3,5-10]}$. Although it seems to have no sex or age predilection, several studies have shown a slightly higher prevalence in women, with an average age at presentation of 30 years ${ }^{[2,4]}$.

There are three types of IPEH: (a) a primary type described by Hashimoto et al., which is a pure or primary endovascular form that originates in dilated vascular spaces (the most common type); (b) a mixed or secondary form that occurs in pre-existing vascular abnormalities, such as varices, haemangiomas and arteriovenous malformations; and (c) the undetermined type, which is a rare extravascular haemangioma form that arises from haematomas ${ }^{[1]}$. The pathogenesis of IPEH remains unclear but appears to be multifactorial ${ }^{[4]}$. Although in $30 \%$ of cases it is related to triggers such as trauma or vascular abnormalities, in the majority of cases the causes are undetermined ${ }^{[5]}$. In the case described, although the histological features of the surrounding adipose tissue were compatible with possible external trauma, the patient denied a history of trauma and it was not known if there were pre-existing vascular malformations, so the authors considered it to be the primary form. Clinical presentation, pattern of growth, and association with other lesions is associated with a benign process, presumably reactive to blood vessel injury or thrombosis ${ }^{[2,5]}$.

The diagnosis of IPEH can be challenging due to vague clinical findings, non-specific imaging characteristics, and its similarity to other benign and malign lesions. Histological characterization plays a critical role in diagnosis and demonstrates a well-circumscribed lesion with papillae fronds with a lining of hyperplastic endothelial cells, as illustrated in our case ${ }^{[3]}$. 
Most importantly, IPEH is often mistaken for angiosarcoma, which is distinguished by its histology, defined by a diffuse infiltrative nature, is never confined to the vascular lumen, has solid, necrotic areas, and demonstrates mitotic figures or pleomorphic cells ${ }^{[3,4]}$. Other differential diagnosis should be considered, including pyogenic granuloma, mucocele, the intravascular form of haemangioma, malignant endovascular papillary angioendothelioma and Kaposi's sarcoma ${ }^{[1]}$.

Surgical excision is the treatment of choice, and generally has a good prognosis. Recurrence is rare and mostly associated with incomplete excision ${ }^{[1,3]}$.

In conclusion, Masson's tumour is a benign, indolent lesion, whose diagnostic importance is linked to its similarity with other pathologies, namely angiosarcoma. The authors highlight the need to recognize these lesions, with timely aetiological study and careful histological characterization for prompt diagnosis.

\section{REFERENCES}

1. Hashimoto H, Daimaru Y, Enjoji M. Intravascular papillary endothelial hyperplasia. A clinicopathologic study of 91 cases. Am J Dermatopathol 1983;5(6):539-546.

2. Kuo T, Sayers CP, Rosai J. Masson's "vegetant intravascular hemangioendothelioma:" a lesion often mistaken for angiosarcoma: study of seventeen cases located in the skin and soft tissues. Cancer 1976;38(3):1227-1236.

3. Pantanowitz L, Muc R, Spanger M, Sonnendecker H, McIntosh WA. Intravascular papillary endothelial hyperplasia (Masson's tumor) manifesting as a lateral neck mass. Ear Nose Throat J 2000;79(10):806, 809-810.

4. Luce EB, Montgomery MT, Redding SW, Aufdemorte TB. Intravascular angiomatosis (Masson's lesion). J Oral Maxillofac Surg 1988;46(9):736-741.

5. Giannitto C, Mercante G, Spriano G, Natoli R, Gaino F, Lofino L, et al. CT and MRI findings of head and neck Masson's tumor: a rare case report and systematic review of the literature. Rep Med Imaging 2021;14:53-64.

6. El Husseiny M, Benhaiem N, Vayssairat M, Allaire E. Masson's vegetant intravascular hemangioendothelioma in an external jugular vein aneurysm with recurrent thrombosis. J Vasc Surg 2011:53(6):1723-1725

7. Alotaibi M, Geisler V, Olze H, Knopke S. Masson tumor as a neck mass. Eur Ann Otorhinolaryngol Head Neck 2020;137(3):217-218.

8. Suh KS, Shin KS, Park IA. Intravascular papillary endothelial hyperplasia of the neck masquerading as malignancy on fine-needle aspiration cytology. Diagn Cytopathol 2003;29(1):14-17.

9. Yücesoy C, Coban G, Yilmazer D, Oztürk E, Hekimoğlu B. Intravascular papillary endothelial hyperplasia (Masson's hemangioma) presenting as a lateral neck mass. JBR-BTR 2009;92(1):20-22

10. Nangia A, Sehgal S, Agarwal K. Masson's tumor masquerading as a papillary adenocarcinoma on fine needle aspiration cytology: a case report. Turk Patoloji Derg 2018;34(2):179181. 